\title{
The Moss Flora of Akdăg Mountain (Amasya, Turkey)
}

\author{
Kerem Canli and Barbaros Çetin \\ Department of Biology, Faculty of Science, Dokuz Eylül University, 35390 Izmir, Turkey \\ Correspondence should be addressed to Kerem Canli; biyoloji@gmail.com
}

Received 31 July 2014; Revised 20 November 2014; Accepted 27 November 2014; Published 23 December 2014

Academic Editor: Calum R. Wilson

Copyright (C) $2014 \mathrm{~K}$. Canli and B. Çetin. This is an open access article distributed under the Creative Commons Attribution License, which permits unrestricted use, distribution, and reproduction in any medium, provided the original work is properly cited.

\begin{abstract}
The moss flora of Akdağ Mountain (Amasya, Turkey) was investigated. At the result of identifications of 1500 moss specimens, collected from the research area, 178 taxa belonging to 69 genera and 26 families were determined. Among them, 94 taxa are new for A3 grid square according to the Turkey grid system which was adopted by Henderson. The location data of Grimmia crinitoleucophaea Cardot and Barbula enderesii Garov. are the first records for Turkey, and Encalypta spathulata Müll. Hal., Schistidium dupretii (Thér.) W. A. Weber, Weissia condensa var. armata (Thér. \& Trab.) M. J. Cano, Ros \& J. Guerra, Tortella bambergeri (Schimp.), Barbula enderesii Garov., Hedwigia ciliata var. leucophaea Bruch \& Schimp., and Campyliadelphus elodes (Lindb.) Kanda are recorded for the second time to the byroflora of Turkey.
\end{abstract}

\section{Introduction}

Turkey, which is in the transition zone of three biogeographical regions, the Mediterranean, European-Siberian, and Irano-Turanian, is one of the richest countries between the Middle East and Europe in terms of biodiversity [1]. Unfortunately, knowledge of the Turkish bryoflora is still far from complete. To date, neither Turkish nor foreign bryologists have visited some regions, especially south-eastern Turkey. However, some recent additions with increasing research activities indicate that quite a number of new discoveries may be expected. In recent years, the studies on moss biodiversity of Turkey have increased and are enriched with many new findings. Some additions to the moss flora of Turkey in the last years include Didymodon tomaculosus (Blockeel) M. F. V. Corley [2], Schistidium sordidum Hagen. [3], Bryoerythrophyllum rubrum (Jur. ex Geh.) P. C. Chen [4], Seligeria donniana (Sm.) Müll. Hal. [5], Conardia compacta (Drumm. ex Mull. Hal.) H. Rob. and Didymodon icmadophilus (Schimp. ex Müll. Hal.) K. Saito [6], Seligeria trifaria (Brid.) Lindb. and Pseudotaxiphyllum elegans (Brid.) Z. Iwats. [7], Dicranella schreberiana (Hedw.) Dixon, Dicranodontium asperulum (Mitt.) Broth., and Campylopus pyriformis (Schultz) Brid. [8], Grimmia anomala Schimp., Pohlia filum (Schimp.) Mårtensson, and Hookeria acutifolia Hook. \& Grev. [9],
Sphagnum contortum K. F. Schultz, Sphagnum fallax (H. Klinggr.) H. Klinggr., Sphagnum magellanicum Brid., Sphagnum rubellum Wilson [10], and Sphagnum molle Sull. [11].

Akdağ (Amasya), chosen as the study area, is located between Central Anatolia and the Black Sea region within the A3 square according to Henderson's [12] grid system (Figures 1 and 2). Although Akdağ Mountain has been named as one of the most important flora regions of Turkey, the moss flora of the mountain has not been studied before.

\section{Materials and Methods}

Samples were collected from 37 stations containing different habitats (Table 1), between 2009 and 2011. All specimens were deposited in the Herbarium of Ankara University (ANK), Faculty of Science, Department of Biology, Ankara.

The specimens were identified using relevant literature [13-37].

\section{Results and Discussion}

The new records for the $\mathrm{A} 3$ grid square are indicated with a single asterisk, the taxa recorded from Turkey for the second time with double asterisks, and the first location data from Turkey with triple asterisks in the floristic list. Station 


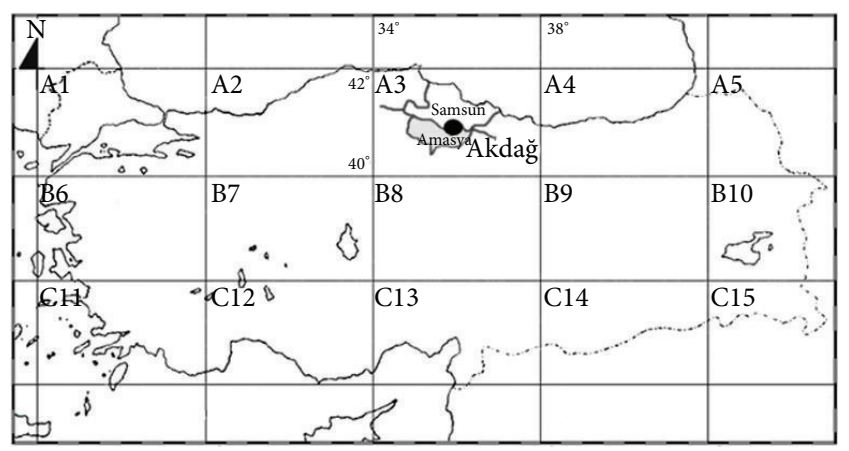

Figure 1: Amasya Akdağ and Henderson's grid system.

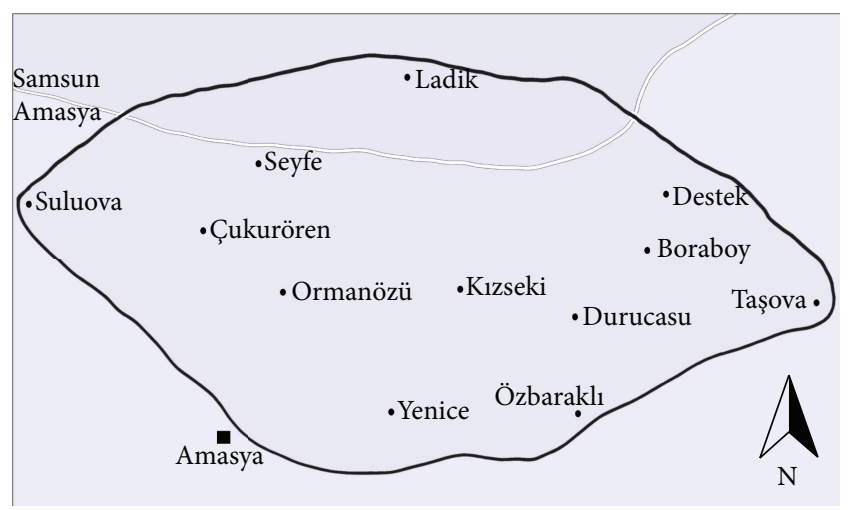

Study area

City border

Figure 2: Study area.

numbers are indicated in parentheses. The bryofloristic list was arranged according to Hill et al. [38] and revised with the latest accepted names according to Ros et al. [39]:

(*) Timmiales (M. Fleisch.) Ochyra

(*) Timmiaceae Schimp.

(*) 1. Timmia Hedw.

(*) 1. Timmia bavarica Hessl. $(20,21)$

Encalyptales Dixon

Encalyptaceae Schimp.

2. Encalypta Hedw.

2. Encalypta streptocarpa Hedw. $(2,16,20,21,26,33)$

(*) 3. E. intermedia Jur. (18)

(*) 4. E. rhaptocarpa Schwägr. (15)

$(*)(* *)$ 5. E. spathulata Müll. Hal. (34)

6. E. vulgaris Hedw. $(26,34)$

Funariales M. Fleisch.

Funariaceae Schwägr.

3. Entosthodon Schwägr.

(*) 7. Entosthodon muhlenbergii (Turner) Fife (2)

\section{Funaria Hedw.}

8. Funaria hygrometrica Hedw. $(1,34)$

Grimmiales M. Fleisch.

Grimmiaceae Arn.

5. Grimmia Hedw.

$(*)(* * *)$ 9. Grimmia crinitoleucophaea Cardot (10, $13,25,34)$

(*) 10. G. funalis (Schwägr.) Bruch \& Schimp. (13)

(*) 11. G. laevigata (Brid.) Brid. $(7,8,13,31,34)$

(*) 12. G. montana Bruch \& Schimp. (25)

(*) 13. G. orbicularis Bruch ex Wilson $(3,7,10,23,33)$

14. G. ovalis (Hedw.) Lindb. (25)

15. G. pulvinata (Hedw.) Sm. (1, 2, 3, 10, 13, 15, 25, 32, $34,35,36)$

16. G. tergestina Tomm. ex Bruch \& Schimp. $(8,25,31)$

17. G. trichophylla Grev. $(3,5,8,36)$

6. Schistidium Bruch \& Schimp.

18. Schistidium apocarpum (Hedw.) Bruch \& Schimp. $(4,13,23,35)$

(*) 19. S. atrofuscum (Schimp.) Limpr. (16)

(*) 20. S. brunnescens Limpr. (17)

(*) 21. S. confertum (Funck) Bruch \& Schimp. (3, 5, 20)

(*) 22. S. crassipilum H. H. Blom (17)

$(*)(* *)$ 23. S. dupretii (Thér.) W. A. Weber (12)

(*) 24. S. elegantulum H. H. Blom $(10,12)$

(*) 25. S. pruinosum (Wilson ex Schimp.) G. Roth (2)

Dicranales H. Philib. ex M. Fleisch

Fissidentaceae Schimp.

\section{Fissidens Hedw.}

26. Fissidens taxifolius Hedw. (12)

\section{Ditrichaceae Limpr.}

8. Ceratodon Brid.

27. Ceratodon purpureus (Hedw.) Brid. (6)

\section{Distichium Bruch \& Schimp.}

28. Distichium capillaceum (Hedw.) Bruch \& Schimp. (2)

\section{Ditrichum Timm ex Hampe}

29. Ditrichum flexicaule (Schwägr.) Hampe $(16,33)$

Dicranaceae Schimp.

11. Dicranella (Müll. Hal.)

30. Dicranella howei Renauld \& Cardot (19)

31. D. varia (Hedw.) Schimp. $(2,20,23)$

12. Dicranum (Müll. Hal.)

32. Dicranum majus Sm. (6) 
TABLE 1: Vegetations and coordinate information of stations.

\begin{tabular}{|c|c|c|c|}
\hline Station & Altitude (m) & Coordinate & Dominant vegetation \\
\hline 1 & 498 & $\mathrm{~N} 40^{\circ} 41.154^{\prime} / \mathrm{E} 035^{\circ} 57,471^{\prime}$ & Pinus nigra subsp. pallasiana \\
\hline 2 & 872 & 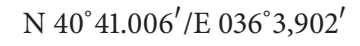 & Quercus robur and Populus tremula \\
\hline 3 & 845 & $\mathrm{~N} 40^{\circ} 53.685^{\prime} / \mathrm{E} 035^{\circ} 46,626^{\prime}$ & Quercus robur and Pinus nigra subsp. pallasiana \\
\hline 4 & 1020 & $\mathrm{~N} 40^{\circ} 54.252^{\prime} / \mathrm{E} 035^{\circ} 52,544^{\prime}$ & Fagus orientalis \\
\hline 5 & 870 & 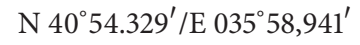 & Reeds and agricultural land \\
\hline 6 & 1070 & 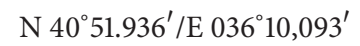 & Pinus sylvestris and Fagus orientalis \\
\hline 7 & 492 & $\mathrm{~N} 40^{\circ} 45.577^{\prime} / \mathrm{E} 036^{\circ} 6,736^{\prime}$ & Pinus brutia \\
\hline 8 & 565 & $\mathrm{~N} 40^{\circ} 44.784^{\prime} / \mathrm{E} 035^{\circ} 59,210^{\prime}$ & Pinus brutia and Quercus hartwissiana \\
\hline 9 & 1330 & 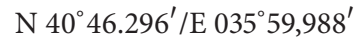 & Juniperus nana and Pinusnigra subsp. pallasiana \\
\hline 10 & 903 & $\mathrm{~N} 40^{\circ} 46.289^{\prime} / \mathrm{E} 036^{\circ} 6,915^{\prime}$ & Juniperus nana and Pinusnigra subsp. pallasiana \\
\hline 11 & 1080 & $\mathrm{~N} 40^{\circ} 50.550^{\prime} / \mathrm{E} 035^{\circ} 47,292^{\prime}$ & Quercus hartwissiana \\
\hline 12 & 1050 & $\mathrm{~N} 40^{\circ} 51.909^{\prime} / \mathrm{E} 035^{\circ} 47,648^{\prime}$ & Pinus nigra subsp. pallasiana \\
\hline 13 & 1100 & 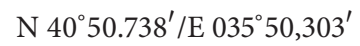 & Pinus nigra subsp. pallasiana \\
\hline 14 & 1430 & $\mathrm{~N} 40^{\circ} 50.507^{\prime} / \mathrm{E} 035^{\circ} 54,296^{\prime}$ & Pinus nigra subsp. pallasiana \\
\hline 15 & 1240 & $\mathrm{~N} 40^{\circ} 48.839^{\prime} / \mathrm{E} 035^{\circ} 57,754^{\prime}$ & Pinus nigra subsp. pallasiana and Astragalus sp. \\
\hline 16 & 1180 & $\mathrm{~N} 40^{\circ} 47.305^{\prime} / \mathrm{E} 036^{\circ} 2,430^{\prime}$ & Pinus nigra subsp. pallasiana and Juniperus oxycedrus \\
\hline 17 & 1750 & 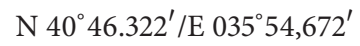 & High mountain meadows \\
\hline 18 & 2040 & $\mathrm{~N} 40^{\circ} 46.786^{\prime} / \mathrm{E} 035^{\circ} 55,621^{\prime}$ & Astragalus sp. and Acantholimon sp. \\
\hline 19 & 227 & $\mathrm{~N} 40^{\circ} 45.304^{\prime} / \mathrm{E} 036^{\circ} 19,371^{\prime}$ & Quercus hartwissiana \\
\hline 20 & 1060 & $\mathrm{~N} 40^{\circ} 48.249^{\prime} / \mathrm{E} 036^{\circ} 9,532^{\prime}$ & Cedrus libani, Pinus nigra subsp. pallasiana, and Fagus orientalis \\
\hline 21 & 1230 & $\mathrm{~N} 40^{\circ} 48.080^{\prime} / \mathrm{E} 036^{\circ} 7,876^{\prime}$ & Cedrus libani and Pinus nigra subsp. pallasiana \\
\hline 22 & 1550 & $\mathrm{~N} 40^{\circ} 47.935^{\prime} / \mathrm{E} 036^{\circ} 6,600^{\prime}$ & Pinus nigra subsp. pallasiana, Astragalus sp., and Euphorbia sp. \\
\hline 23 & 1650 & 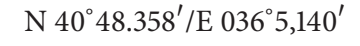 & Pinus nigra subsp. Pallasiana \\
\hline 24 & 1320 & $\mathrm{~N} 40^{\circ} 47.520^{\prime} / \mathrm{E} 036^{\circ} 7,948^{\prime}$ & Pinus nigra subsp. pallasiana, Euphorbia sp., and Juniperus nana \\
\hline 25 & 664 & $\mathrm{~N} 40^{\circ} 46.043^{\prime} / \mathrm{E} 036^{\circ} 7,207^{\prime}$ & Pinus brutia and Quercus hartwissiana \\
\hline 26 & 1320 & $\mathrm{~N} 40^{\circ} 50.614^{\prime} / \mathrm{E} 035^{\circ} 53,952^{\prime}$ & Salix alba \\
\hline 27 & 1570 & 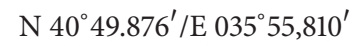 & Astragalus sp. and Acantholimon sp. \\
\hline 28 & 712 & $\mathrm{~N} 40^{\circ} 51.319^{\prime} / \mathrm{E} 036^{\circ} 9,434^{\prime}$ & Pinus sylvestris and Fagus orientalis \\
\hline 29 & 943 & $\mathrm{~N} 40^{\circ} 52.037^{\prime} / \mathrm{E} 036^{\circ} 5,651^{\prime}$ & Carpinus betulus \\
\hline 30 & 1020 & 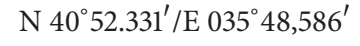 & Pinus sylvestris and Fagus orientalis \\
\hline 31 & 483 & $\mathrm{~N} 40^{\circ} 45.227^{\prime} / \mathrm{E} 035^{\circ} 36,509^{\prime}$ & Agricultural land \\
\hline 32 & 905 & 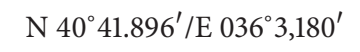 & Pinus brutia and Quercus hartwissiana \\
\hline 33 & 1110 & $\mathrm{~N} 40^{\circ} 38.948^{\prime} / \mathrm{E} 035^{\circ} 57,307^{\prime}$ & Pinus nigra subsp. pallasiana \\
\hline 34 & 513 & 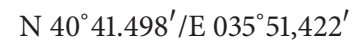 & Pinus nigra subsp. pallasiana \\
\hline 35 & 1520 & $\mathrm{~N} 40^{\circ} 52.581^{\prime} / \mathrm{E} 035^{\circ} 54,388^{\prime}$ & Pinus nigra subsp. pallasiana \\
\hline 36 & 1210 & $\mathrm{~N} 40^{\circ} 45.033^{\prime} /$ E $035^{\circ} 52,059^{\prime}$ & Quercus hartwissiana \\
\hline 37 & 1890 & 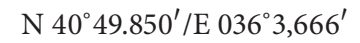 & Astragalus sp. and Acantholimon sp. \\
\hline
\end{tabular}

(*) 33. D. polysetum Sw. ex anon. (12)

34. D. tauricum Sapjegin $(9,23,27)$

Pottiales M. Fleisch

Pottiaceae Schimp.

13. Eucladium Bruch \& Schimp.

35. Eucladium verticillatum (With.) Bruch \& Schimp. $(1,2)$

\section{Gymnostomum Nees \& Hornsch.}

(*) 36. Gymnostomum aeruginosum Sm. (2)

(*) 15. Gyroweisia Schimp.
(*) 37. Gyroweisia tenuis (Hedw.) Schimp. (2)

16. Tortella (Müll. Hal.) Limpr.

$(*)(* *)$ 38. Tortella bambergeri (Schimp.) Broth. (22)

(*) 39. T. humilis (Hedw.) Jenn. $(9,12)$

40. T. inclinata (R. Hedw.) Limpr. (16)

41. T. squarrosa (Brid.) Lindb. (2)

42. T. tortuosa (Hedw.) Limpr (2, 6, 9, 12, 16, 20, 23, 37)

\section{Trichostomum Bruch}

43. Trichostomum brachydontium Bruch $(20,31)$ 
18. Weissia Hedw.

(*) $(* *)$ 44. Weissia condensa var. armata (Thér. \& Trab.) M. J. Cano, Ros \& J. Guerra (10)

45. W. controversa var. controversa Hedw. (33)

19. Barbula Hedw.

46. Barbula convoluta var. convoluta Hedw. $(1,12,19)$

(*) 47. B. convoluta var. sardoa Schimp. $(1,12)$

$(*)(* * *) 48$. B. enderesii Garov. (21)

49. B. unguiculata Hedw. $(1,2,5,28)$

\section{Bryoerythrophyllum P. C. Chen}

50. Bryoerythrophyllum recurvirostrum (Hedw.) P. C. Chen $(2,26)$

(*) 51. B. rubrum (Jur. ex Geh.) P. C. Chen (21)

(*) 21. Cinclidotus P. Beauv.

(*) 52. Cinclidotus danubicus Schiffn. \& Baumgartner (2)

(*) 53. C. riparius (Host ex Brid.) Arn. (2)

22. Crossidium Jur.

54. Crossidium squamiferum (Viv.) Jur. (28)

23. Didymodon Hedw.

55. Didymodon acutus (Brid.) K. Saito $(2,7,8)$

56. D. fallax (Hedw.) R. H. Zander (1)

(*) 57. D. ferrugineus (Schimp. ex Besch.) M. O. Hill $(3,12)$

(*) 58. D. luridus Hornsch. $(12,33)$

59. D. nicholsonii Culm. $(12,33)$

(*) 60. D. rigidulus Hedw. (8)

(*) 61. D. tomaculosus (Blockeel) M. F. V. Corley (20) published as Asia record (Canlı \& Çetin, 2012)

(*) 62. D. tophaceus (Brid.) Lisa $(1,35)$

63. D. vinealis (Brid.) R. H. Zander $(1,21,34)$

\section{Pseudocrossidium R. S. Williams}

(*) 64. Pseudocrossidium hornschuchianum (Schultz) R. H. Zander $(5,34)$

(*) 65. P. revolutum (Brid.) R. H. Zander (34)

25. Syntrichia Brid.

(*) 66. Syntrichia calcicola J. J. Amann $(13,22,25)$

67. S. caninervis Mitt. (8)

(*) 68. S. laevipila Brid. (8)

(*) 69. S. montana Nees $(1,2,8,13)$

70. S. norvegica F. Weber (17)

(*) 71. S. ruralis var. ruraliformis (Besch.) Delogne $(14,26,27,33,35)$

72. S. ruralis var. ruralis (Hedw.) F. Weber \& D. Mohr (3, 17, 23, 27, 34, 36, 37)

73. S. virescens (De Not.) Ochyra (2)

\section{Tortula Hedw.}

(*) 74. Tortula brevissima Schiffn. (2)

(*) 75. T. canescens Mont. (37)

76. T. inermis (Brid.) Mont. $(2,10)$

(*) 77. T. lindbergii Broth. (2)

78. T. muralis Hedw. $(2,3,15,17,21,32)$

(*) 79. T. schimperi M. J. Cano, O. Werner \& J. Guerra $(21,23,29,37)$

80. T. subulata Hedw. $(9,12,21,37)$

(*) 81. T. vahliana (Schultz) Mont. $(11,28)$

\section{Orthotrichales Dixon}

Orthotrichaceae Arn.

\section{Orthotrichum Hedw.}

82. Orthotrichum anomalum Hedw. $(2,8,26,33)$

(*) 83. O. cupulatum Hoffm. ex Brid. $(2,9,12)$

84. O. urnigerum Myrin $(2,33)$

85. O. diaphanum Schrad. ex Brid. $(2,33)$

86. O. pallens Bruch ex Brid. $(2,8,11,12)$

(*) 87. O. rupestre Schleich. ex Schwägr. (36)

88. O. affine Schrad. ex Brid (2)

(*) 89. O. speciosum Nees $(3,11,12)$

90. O. striatum Hedw. (3)

\section{Hedwigiales Ochyra}

(*) Hedwigiaceae Schimp.

(*) 28. Hedwigia P. Beauv.

(*) (**) 91. Hedwigia ciliata var. leucophaea Bruch \& Schimp. (3)

Bryales Limpr.

Bartramiaceae Schwägr.

29. Philonotis Brid.

(*) 92. Philonotis caespitosa Jur. (22)

93. P. fontana (Hedw.) Brid. (15)

(*) 94. P. seriata Mitt. (17)

Bryaceae Schwägr.

30. Bryum Hedw.

95. Bryum argenteum Hedw. $(12,17,37)$

(*) 96. B. funkii Schwägr. (13)

(*) 97. B. schleicheri DC. (22)

(*) 98. B. subapiculatum Hampe (20)

(*) 99. B. weigelii Spreng. (22)

\section{Imbribryum N. Pedersen}

100. Imbribryum alpinum Huds. ex With. (11)

(*) 101. I. mildeanum (Jur.) J. R. Spence (1)

32. Ptychostomum Hornsch. 
*) 102. Ptychostomum borreale (F. Weber \& D. Mohr) Ochyra \& Bednarek-Ochyra (2)

103. P. capillare (Hedw.) Holyoak \& N. Pedersen (12, 20)

(*) 104. P. cernuum (Hedw.) Hornsch. (2)

(*) 105. P. creberrimum (Taylor) J. R. Spence \& H. P. Ramsay (21)

106. Ptychostomum imbricatulum (Müll. Hal.) Holyoak \& N. Pedersen (20)

(*) 107. P. moravicum (Podp.) Ros \& Mazimpaka (8, $9,20)$

108. P. pallens (Sw.) J. R. Spence $(1,12,15)$

109. P. pseudotriquetrum (Hedw.) J. R. Spence \& H. P. Ramsay (3)

Mielichhoferiaceae Schimp.

33. Pohlia Hedw.

110. Pohlia cruda (Hedw.) Lindb. (20)

(*) 111. P. ludwigii (Spreng. ex Schwägr.) Broth. (27)

Mniaceae Schwägr.

34. Mnium Hedw.

(*) 112. Mnium stellare Hedw. (20)

Cinclidiaceae Kindb.

35. Rhizomnium (Broth.) T. J. Kop.

113. Rhizomnium punctatum (Hedw.) T. J. Kop. (13)

Plagiomniaceae T. J. Kop.

36. Plagiomnium T. J. Kop.

(*) 114. Plagiomnium affine (Blandow ex Funck) T. J. Kop. (12)

115. P. elatum (Bruch \& Schimp.) T. J. Kop. (2)

(*) 116. P. medium (Bruch \& Schimp.) T. J. Kop. (2)

Hypnales (M. Fleisch.) W. R. Buck \& Vitt

(*) Fontinalaceae Schimp.

(*) 37. Fontinalis Hedw.

(*) 117. Fontinalis antipyretica subsp. antipyretica Hedw. (2)

(*) 118. F. antipyretica subsp. gracilis (Lindb.) Kindb.

(2)

(*) 119. F. hypnoides var. hypnoides C. Hartm. (2)

Amblystegiaceae Kindb.

38. Amblystegium Schimp.

120. Amblystegium serpens (Hedw.) Schimp. $(1,2)$

(*) 39. Campyliadelphus (Kindb.) R. S. Chopra

(*) 121. Campyliadelphus chrysophyllus (Brid.) R. S. Chopra (12)

(*) 122. C. elodes (Lindb.) Kanda (1)

40. Cratoneuron (Sull.) Spruce
123. Cratoneuron filicinum (Hedw.) Spruce (2, 13, 17, $21,22)$

\section{Hygroamblystegium Loeske}

124. Hygroamblystegium fluviatile (Hedw.) Loeske (1)

(*) 125. H. tenax (Hedw.) Jenn. (2)

126. H. varium (Hedw.) Mönk. (15)

(*) 127. H. varium var. humile (P. Beauv.) Vanderp. \& Hedenäs $(1,2,12)$

(*) 42. Hygrohypnum Lindb.

(*) 128. Hygrohypnum luridum (Hedw.) Jenn. (22)

43. Palustriella Ochyra

129. Palustriella commutata (Hedw.) Ochyra (2, 15, 37)

(*) 130. P. decipiens (De Not.) Ochyra (2)

131. P. falcata (Brid.) Hedenäs (2)

44. Pseudocampylium Vanderp. \& Hedenäs

(*) 132. Pseudocampylium radicale (P. Beauv.) Schimp. $(2,4)$

Leskeaceae Schimp.

45 Pseudoleskeella Kindb.

133. Pseudoleskeella catenulata (Brid. ex Schrad.) Kindb (4)

(*) 134. P. nervosa (Brid.) Nyholm (4)

(*) 135. P. tectorum (Funck ex Brid.) Kindb. ex Broth. (9)

\section{Thuidiaceae Schimp.}

46. Abietinella Kindb.

136. Abietinella abietina var. abietina (Hedw.) M. Fleisch. (2)

137. A. abietina var. hystricosa (Mitt.) Sakura $(3,12)$

47. Thuidium Schimp.

138. Thuidium delicatulum (Hedw.) Schimp. (35)

Brachytheciaceae Schimp.

48. Pseudoscleropodium Schimp.

139. Pseudoscleropodium purum (Hedw.) M. Fleisch. (12)

49. Plasteurhynchium M. Fleisch. ex Broth.

(*) 140. Plasteurhynchium striatulum (Spruce) M. Fleisch. (13)

50. Rhynchostegium Schimp.

141. Rhynchostegium alopecuroides (Brid.) A. J. E. Sm. $(13,15)$

142. R. confertum (Dicks.) Schimp. (31)

(*) 143. R. megapolitanum (Blandow ex F. Weber \& D. Mohr) Schimp. (1)

144. R. riparioides (Hedw.) Cardot $(1,2)$

51. Oxyrrhynchium (Schimp.) Warnst 
145. Oxyrrhynchium hians (Hedw.) Loeske (13)

(*) 146. O. speciosum (Brid.) Warnst. $(2,12)$

\section{Kindbergia Ochyra}

147. Kindbergia praelonga (Hedw.) Ochyra $(9,11,12)$

53. Sciuro-hypnum Hampe

(*) 148. Sciuro-hypnum latifolium Ignatov \& Huttunen $(2,15,17)$

(*) 149. S. reflexum (Starke) Ignatov \& Huttunen (11)

54. Brachythecium Schimp.

150. Brachythecium albicans (Hedw.) Schimp. $(11,19)$

(*) 151. B. geheebii Milde $(4,11)$

152. B. glareosum (Bruch ex Spruce) Schimp. (19)

153. B. rivulare Schimp. (2)

\section{Eurhynchiastrum Ignatov \& Huttunen}

154. Eurhynchiastrum pulchellum (Hedw.) Ignatov \& Huttunen $(20,23)$

\section{Brachytheciastrum Ignatov \& Huttunen}

155. Brachytheciastrum velutinum (Hedw.) Ignatov \& Huttunen $(20,21)$

\section{Homalothecium Schimp.}

(*) 156. Homalothecium aureum (Spruce) H. Rob. (5)

157. H. lutescens (Hedw.) H. Rob. $(3,12,14,28,35)$

158. H. philippeanum (Spruce) Schimp. $(21,30)$

159. H. sericeum (Hedw.) Schimp. (1, 2, 13, 20, 23)

Hypnaceae Schimp.

58. Calliergonella Loeske

160. Calliergonella cuspidata (Hedw.) Loeske $(15,17)$

(*) 59. Campylophyllum (Schimp.) M. Fleisch.

(*) 161. Campylophyllum sommerfeltii (Myrin) Hedenäs (2)

60. Homomallium (Schimp.) Loeske

162. Homomallium incurvatum (Schrad. ex Brid.) Loeske (4)

\section{Hypnum Hedw.}

163. Hypnum andoi A. J. E. Sm. (3, 6, 7, 11, 12, 20)

(*) 164. H. bambergeri Schimp. (14)

165. H. cupressiforme var. cupressiforme Hedw. (2, 20, 24)

166. H. cupressiforme var. lacunosum Brid. $(9,34)$

(*) 167. H. cupressiforme var. subjulaceum Molendo (14)

168. H. imponens Hedw. (12)

(*) 169. H. recurvatum (Lindb. \& Arnell) Kindb. (26)

(*) 170. H. revolutum (Mitt.) Lindb. (20)

62. Pylaisia Schimp.

171. Pylaisia polyantha (Hedw.) Schimp. (9)
(*) 63. Taxiphyllum M. Fleisch.

(*) 172. Taxiphyllum wissgrillii (Garov.) Wijk \& Margad. (20)

Pterigynandraceae Schimp.

64. Heterocladium Schimp.

(*)173. Heterocladium dimorphum (Brid.) Schimp. (9)

65. Pterigynandrum Hedw.

174. Pterigynandrum filiforme Hedw. $(20,21,22)$

(*) Pylaisiadelphaceae Goffinet \& W. R. Buck

(*) 66. Platygyrium Schimp.

(*) 175. Platygyrium repens (Brid.) Schimp. (20)

(*) Cryphaeaceae Schimp.

(*) 67. Cryphaea D. Mohr

(*) 176. Cryphaea heteromalla (Hedw.) D. Mohr (4)

Leucodontaceae Schimp.

68. Leucodon Schwägr.

177. Leucodon sciuroides (Hedw.) Schwägr. (4, 8, 20, 23, 30)

Neckeraceae Schimp.

69. Neckera Hedw.

(*) 178. Neckera menziesii Drumm. $(23,30)$

A total of 178 taxa belonging to 69 genera and 26 families were determined following the identification of 1500 specimens collected from 37 localities between 2009 and 2011. Of these, 94 taxa are new for the A3 square. The location data of Grimmia crinitoleucophaea Cardot and Barbula enderesii Garov. are the first records for Turkey, and Encalypta spathulata Müll. Hal., Schistidium dupretii (Thér.) W. A. Weber, Weissia condensa var. armata (Thér. \& Trab.) M. J. Cano, Ros \& J. Guerra, Tortella bambergeri (Schimp.), Barbula enderesii Garov., Hedwigia ciliata var. leucophaea Bruch \& Schimp., and Campyliadelphus elodes (Lindb.) Kanda are recorded for the second time.

Grimmia crinitoleucophaea Cardot. The basal marginal cells of the perichaetial and subperichaetial leaves are hyaline. Setae are very short and sporophytes are hidden between perichaetial leaves.

Encalypta spathulata Müll. Hal. Plants form an extensive mat and are covered with a mass of pale-colored calyptrae. The rostrum of the calyptra is short. Seta is red to dark red and quite fragile. Leaves are narrow and irregularly twisted, with a shiny dark-brown costa.

Barbula enderesii Garov. Plants have strongly differentiated convolute perichaetial leaves. Setae are mostly yellow and annulus is strongly differentiated. Leaves are strongly falcate with dense high papillae.

Schistidium dupretii (Thér.) W. A. Weber. Plants are small and form low tufts. The central strand is distinct and hair-point is 
TABLE 2: The comparison of the taxa distribution according to the families.

\begin{tabular}{lccccc}
\hline & $\begin{array}{c}\text { Amasya } \\
\text { Akdağ }\end{array}$ & $\begin{array}{c}\text { Cankırı Gürgenli } \\
\text { Mountain }\end{array}$ & $\begin{array}{c}\text { Ilgaz Yenice } \\
\text { Forests }\end{array}$ & $\begin{array}{c}\text { Ilgaz Mountain } \\
\text { National Park }\end{array}$ & $\begin{array}{c}\text { Çankırı Eldivan } \\
\text { Mountain }\end{array}$ \\
\hline Pottiaceae & 27 & 16,9 & 15,6 & 13,7 & 23,3 \\
Brachytheciaceae & 11,6 & 20,5 & 12,1 & 13,7 & 18,3 \\
Grimmiaceae & 9,4 & 7,2 & 10 & 6,4 & 8,3 \\
\hline
\end{tabular}

very short. Costa and leaf margins are smooth. Sporophytes are common. Peristome teeth are red and entire.

Weissia condensa var. armata (Thér. \& Trab.) M. J. Cano, Ros \& J. Guerra. Plants are almost $0.5 \mathrm{~cm}$. Leaves are patent and margins are slightly incurved. Upper laminal cells are slightly papillose.

Hedwigia ciliata var. leucophaea Bruch \& Schimp. Differs from $H$. ciliata var. ciliata in the length of the hair-point (7-33\% versus $22-65 \%$ of leaf length), in having less strongly papillose hair-points, and in having more strongly recurved stem leaf margins.

Tortella bambergeri (Schimp.). The long, narrow leaves are slightly curved or almost straight when moist and dry to a contorted spiral. They have plane margins. The upper part of the leaf is very fragile and most stems in a tuft have only a few leaf tips present. Transverse section of stem has a distinct central strand.

Campyliadelphus elodes (Lindb.) Kanda. The plants are rather slender, with irregularly and rather loosely branched, dull green or yellow-green shoots. Shoots reach $4-5 \mathrm{~cm}$ or more in length. Leaves are acute and gradually tapering from base to apex. Leaf margins are obscurely denticulate above. Costa is long and single and is usually extending the apex or near the apex.

The dominant family in the study area is Pottiaceae (48 taxa). Other families with the highest number of taxa are, respectively, Brachytheciaceae (21), Grimmiaceae (17), and Bryaceae (15). The most species-rich genera recorded were Grimmia (9), Didymodon (9), Tortula (9), and Orthotrichum (9). Acrocarpous mosses constitute $65 \%$ and pleurocarpousmosses constitute $35 \%$.

The data from this survey was compared to studies from neighboring areas (Table 2) [40-43] and shows that the number of taxa of Pottiaceae family members, which display acrocarpous mosses in the Mediterranean, is higher than from other regions. These findings are similar to seed plant vegetation surveys of the area. This is due to the wet and mild climate, large number of microhabitats and enclaves, and different ecological conditions present in the region.

It is hoped that further studies will contribute more species to the knowledge of moss flora of Turkey and that this study will be useful as a guide for future research.

\section{Conflict of Interests}

The authors declare that there is no conflict of interests regarding the publication of this paper.

\section{Acknowledgments}

Special thanks are due to Diana Horton, Jesus Munoz, Bruce Allen, and Michael Lueth for confirming some determinations.

\section{References}

[1] Z. Kaya and D. J. Raynal, "Biodiversity and conservation of Turkish forests," Biological Conservation, vol. 97, no. 2, pp. 131141, 2001.

[2] K. Canli and B. Çetin, "Didymodon tomaculosus (Blockeel) M.F.V. Corley, new to the moss flora of Turkey and Asia," Bangladesh Journal of Botany, vol. 41, no. 2, pp. 177-179, 2012.

[3] N. Batan, M. Alataş, and T. Özdemir, "Leptoscyphus cuneifolius (Lophocoleaceae, Marchantiophyta) new to Southwest Asia," Cryptogamie, Bryologie, vol. 34, no. 3, pp. 373-377, 2013.

[4] N. Batan and T. Özdemir, "Bryoerythrophyllum rubrum (Pottiaceae)-a new moss in the Turkish bryophyte flora," Phytologia Balcanica, vol. 18, pp. 117-120, 2012.

[5] S. Ursavas and B. Cetin, "Contribution to the Moss Flora of Kizildağ (Isparta) National Park in Turkey," Current Progress in Biological Research, pp. 41-70, 2013.

[6] M. Kirmaci, H. Kurschner, and A. Erdaô, "New and noteworthy records to the bryophyte flora of turkey and southwest asia," Cryptogamie, Bryologie, vol. 33, no. 3, pp. 267-270, 2012.

[7] Ö. Ören, G. Uyar, and T. Keçel, "The bryophyte flora of the western part of the Küre Mountains (Bartin, Kastamonu), Turkey," Turkish Journal of Botany, vol. 36, no. 5, pp. 538-557, 2012.

[8] N. Batan and T. Özdemir, "Bryoflora of Dernekpazarı district of Trabzon (Turkey) province," Phytologia Balcanica, vol. 6, no. 2, pp. 45-49, 2013.

[9] G. Uyar and M. Ören, "Three remarkable new moss records for South-West Asia from Northern Turkey," Turkish Journal of Botany, vol. 37, no. 2, pp. 363-368, 2013.

[10] M. Kirmaci and H. Kürschner, "The genus Sphagnum L. in Turkey - With S. contortum, S. fallax, S. magellanicum and S. rubellum, new to Turkey and Southwest Asia," Nova Hedwigia, vol. 96, no. 3-4, pp. 383-397, 2013.

[11] G. Abay and T. Keçeli, "Sphagnum molle (Sphagnaceae, Bryophyta) in Turkey and SW Asia," Cryptogamie, Bryologie, vol. 35, no. 1, pp. 105-112, 2014. 
[12] D. M. Henderson, Contributions to the Bryophyte Flora of Turkey: IV. Notes from the Royal Botanic Garden Edinburgh, vol. 23, 1961.

[13] R. H. Zander, "Genera of the pottiaceae: mosses of harsh environments," Bullettin of the Buffalo Society of Naturel Sciences, vol. 32, pp. 1-378, 1993.

[14] W. Frey, J. P. Frahm, E. Fischer, and W. Lobin, Kleine Kryptogamenflora, Die Moos-und Farnpflanzen Europas, Gustav Fischer, Stuttgart, Germany, 1995 (German).

[15] H. C. Greven, Grimmia Hedw. (Grimmiacea, Musci) in Europe, Backhuys, Leiden, the Netherlands, 1995.

[16] E. Nyholm, Illustrated Flora of Nordic Mosses, Fasc. 4, Nordic Bryological Society, Lund, Sweden, 1998.

[17] C. C. Pedrotti, Flora Dei Muschi d'Italia, Sphagnopsida, Andreaopsida, Bryopsida (I parte), Antonia Delfino, Rome, Italy, 2001, (Italian).

[18] H. C. Greven, Grimmias of the World, Backhuys Publishers, Leiden, The Netherlands, 2003.

[19] C. C. Heyn and I. Herrnstadt, Israel Academy of Sciences and Humanities, Israel Academy of Sciences and Humanities, Jerusalem, Israel, 2004.

[20] A. J. E. Smith, The Moss Flora of Britain, Cambridge University Press, Cambridge, UK, 2nd edition, 2004.

[21] C. Cortini Pedrotti, Flora dei Muschi d'Italia, Bryopsida (II Parte), Antonia Delfino Editore, Rome, Italy, 2006, (Italian).

[22] W. Frey, J. P. Frahm, E. Fischer, and W. Lobin, The Liverworts, Mosses and Ferns of Europe, English edition revised and edited by T. L. Blockeel, Harley Books, Colchester, UK, 2006.

[23] J. Guerra, M. J. Cano, and R. M. Ros, Flora Briofítica Ibérica, vol. 3, Sociedad Espanola de Briologia, Murcia, Spain, 2006, (Spanish).

[24] M. Brugues, R. M. Cros, and J. Guerra, Flora Briofítica Ibérica, vol. 1, Sociedad Espanola de Briologia, Murcia, Spain, 2007, (Spanish).

[25] D. G. Horton, "A revision of the Encalyptaceae (Musci), with particular reference to the North American taxa. Part I," Journal-Hattori Botanical Laboratory, vol. 53, pp. 365-418, 1982.

[26] D. G. Horton, "A revision of the Encalyptaceae (Musci), with particular reference to the North American taxa. Part II," Journal of the Hattori Botanical Laboratory, vol. 54, pp. 353-532, 1983.

[27] J. Munoz and F. Pando, A World Synopsis of the Genus Grimmia (Musci, Grimmiaceae), vol. 83 of Monographs in Systematic Botany from the Missouri Botanical Garden, 2000.

[28] B. Bremer, "A taxonomic revision of Schistidium (Grimmiaceae, Bryophyta) 1," Lindbergia, vol. 6, pp. 1-16, 1980.

[29] B. Bremer, "A taxonomic revision of Schistidium (Grimmiaceae, Bryophyta) 2," Lindbergia, vol. 6, pp. 89-117, 1980.

[30] B. Bremer, "A taxonomic revision of Schistidium (Grimmiaceae, Bryophyta) 3," Lindbergia, vol. 7, pp. 73-90, 1981.

[31] C. Casas, M. Brugues, R. M. Cros, and C. Sérgio, Handbook of Mosses of the Iberian Peninsula and the Balearic Islands, Institut d'Estudis Catalans, Barcelona, Spain, 2007.

[32] G. Chien, R. C. Marshall, and H. Si, Moss Flora of China, English Version, Volume 1. Sphagnaceae-Leucobryaceae, Missouri Botanical Garden, 1999.

[33] G. Chien, Moss Flora of China, English Version, Volume 3: Grimmiaceae-Tetraphidaceae, Missouri Botanical Garden, 2003.

[34] X. J. Li, R. C. Marshall, and H. Si, Moss Flora of China: BryaceaeTimmiaceae, vol. 4, Missouri Botanical Garden, 2007.
[35] X. J. Li, Moss Flora of China, Volume 2: FissidentaceaePtychomitriaceae, English Version, Missouri Botanical Garden, 2001.

[36] W. Peng-cheng, L. Bang-juan, G. Chien et al., Moss Flora of China: Hookeriaceae-Tuhuidiaceae, vol. 6, Missouri Botanical Garden, 2002.

[37] W. You-Fang, H. Ren-Liang, W. Yu-Huan et al., Moss Flora of China, Volume 7: Amblystegiaceae-Plagiotheciaceae, English Version, Missouri Botanical Garden, 2008.

[38] M. O. Hill, N. Bell, M. A. Bruggeman-Nannenga et al., "An annotated checklist of the mosses of Europe and Macaronesia," Journal of Bryology, vol. 28, no. 3, pp. 198-267, 2006.

[39] R. M. Ros, V. Mazimpaka, U. Abou-Salama et al., "Mosses of the mediterranean, an annotated checklist," Cryptogamie, Bryologie, vol. 34, no. 2, pp. 99-283, 2013.

[40] A. Sahin and G. Abay, "Gürgenli Dağı Karayosunu (Musci) Florasına Katkılar (Bayramören/Çankırı)," Artvin Çoruh Üniversitesi Orman Fakültesi Dergisi, Cilt, vol. 10, no. 2, pp. 83-93, 2009.

[41] S. Ursavas and G. Abay, "Contributions to the bryoflora of Ilgaz Mountains, Yenice Forests, Turkey," Biological Diverity and Conversation, vol. 2, no. 3, pp. 112-121, 2009.

[42] G. Abay and B. Cetin, "The moss flora (Musci) of Ilgaz Mountain National Park," Turkish Journal of Botany, vol. 27, no. 4, pp. 321-332, 2003.

[43] T. Keceli and B. Cetin, "The moss flora of Çankırı-Eldivan mountain," Turkish Journal of Botany, vol. 24, no. 4, pp. 249258,2000 . 

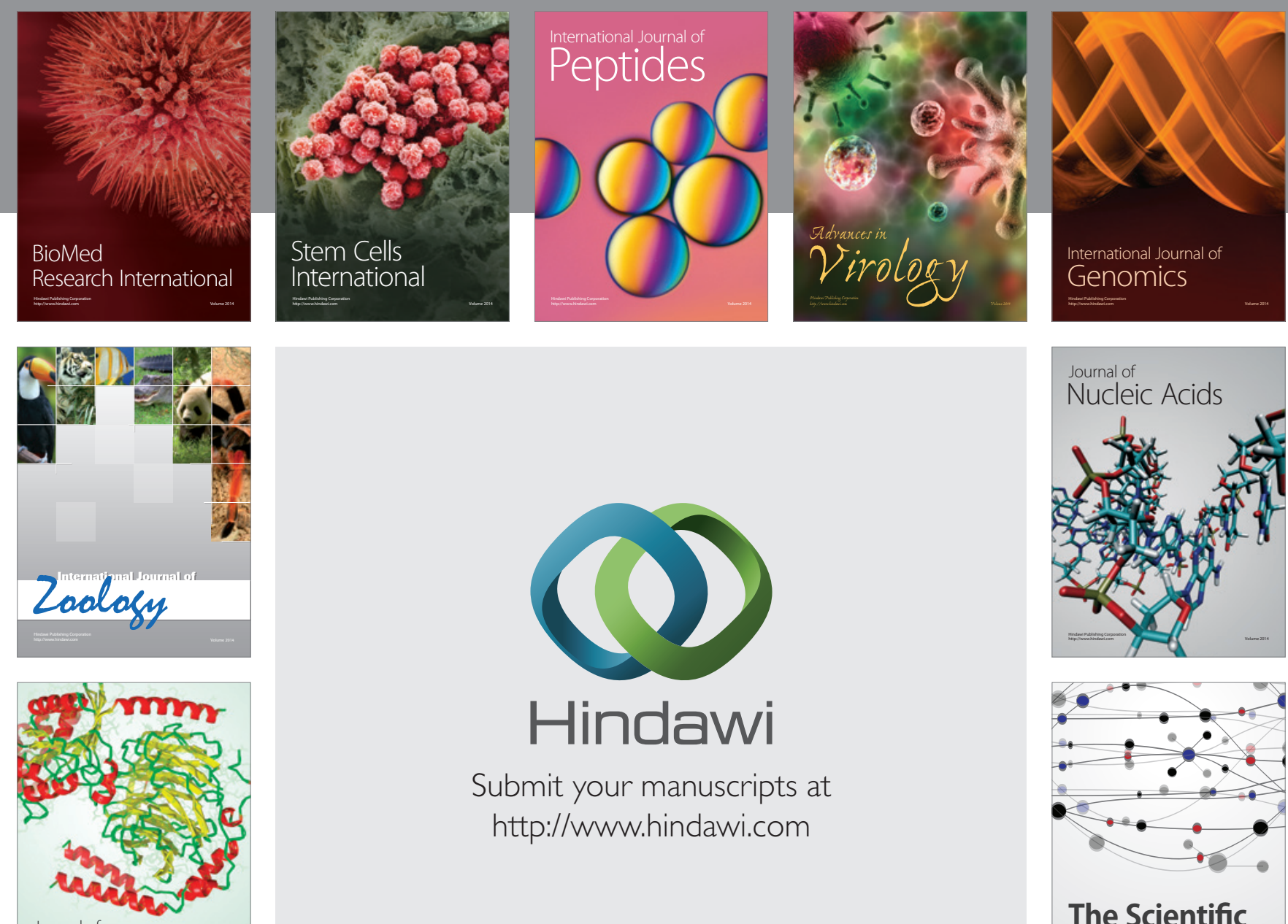

Submit your manuscripts at

http://www.hindawi.com

Journal of
Signal Transduction
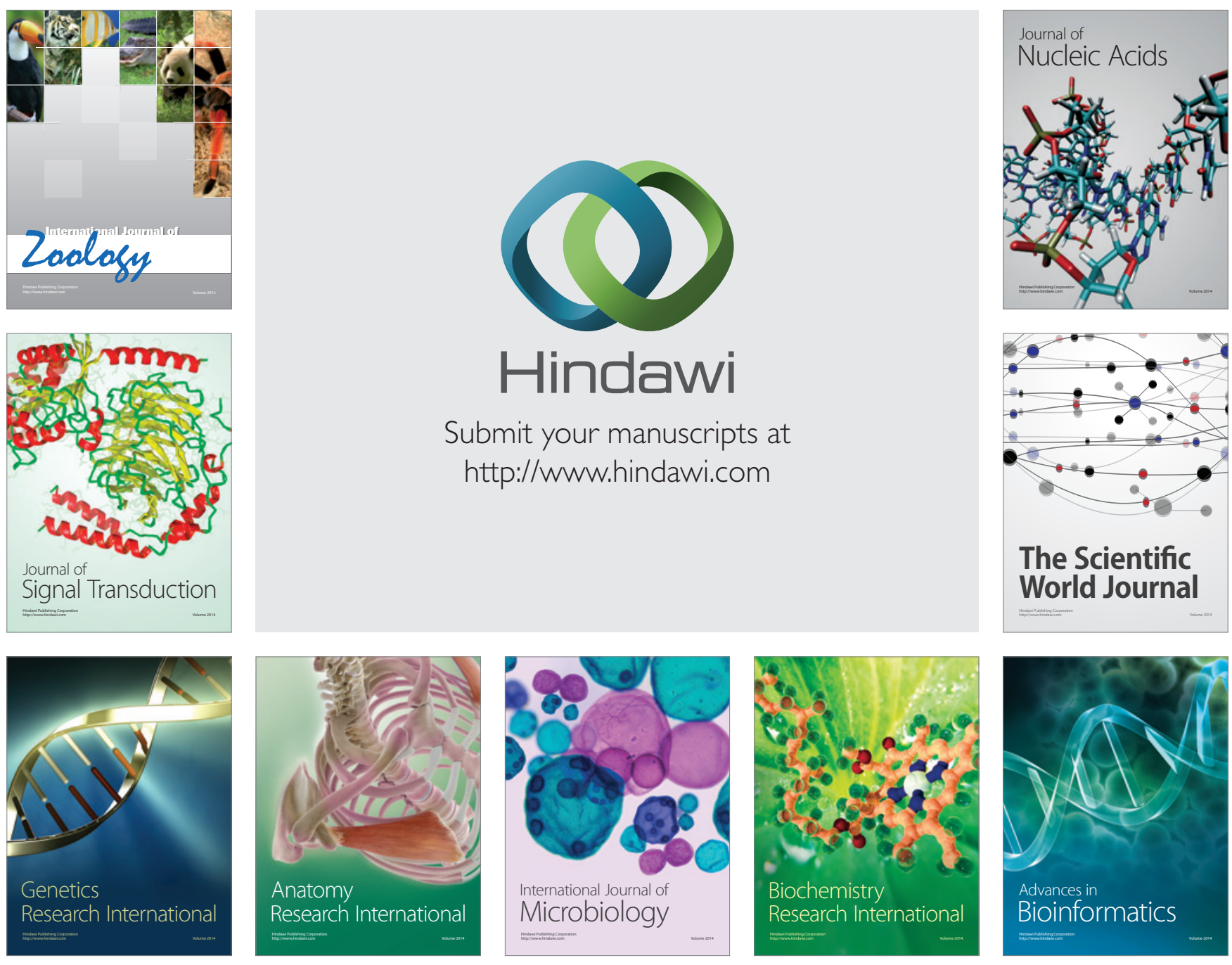

The Scientific World Journal
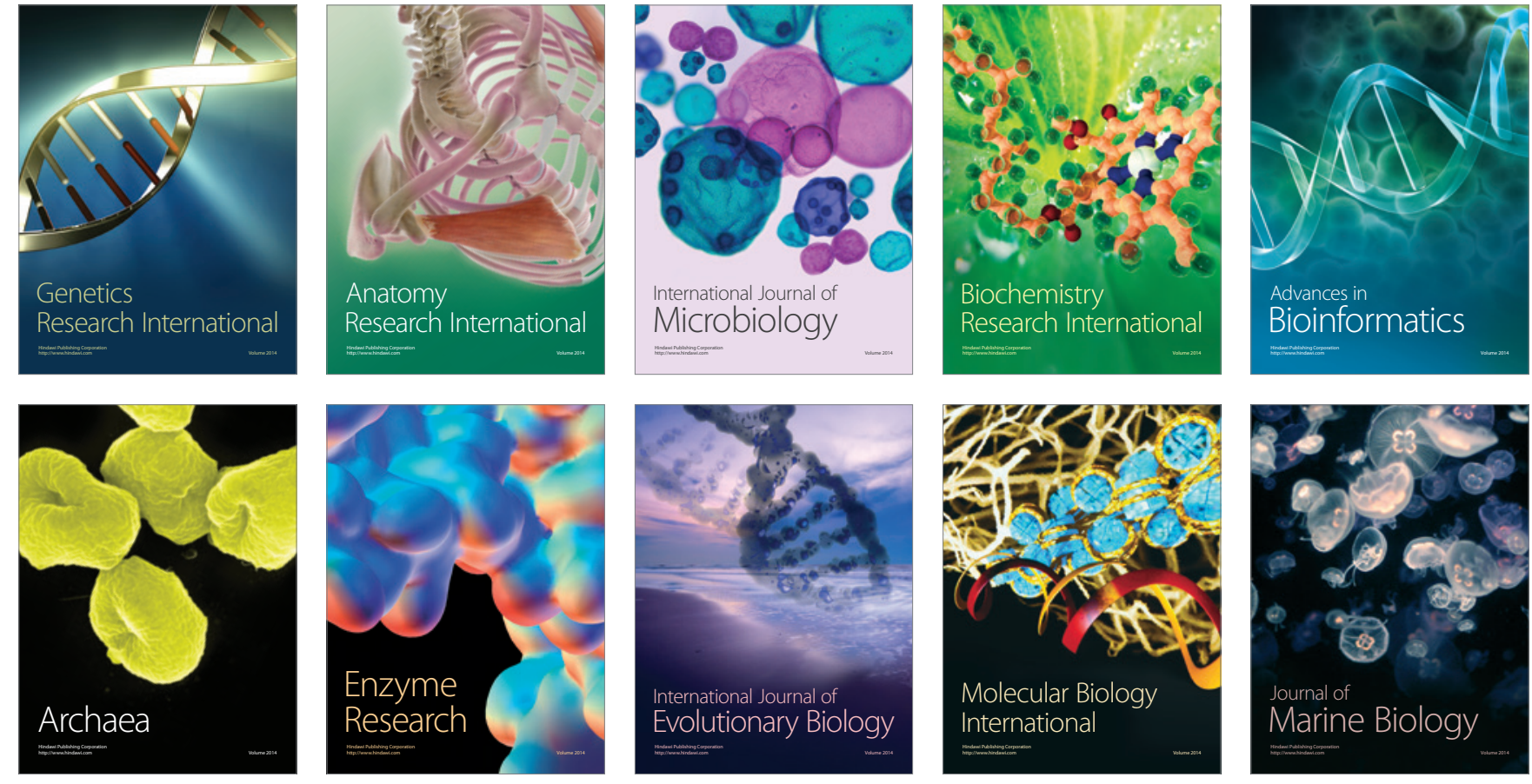